\title{
Evaluation of Semicircular Canal Functions by the Video Head Impulse Test (vHIT) in Individuals with Motion Sickness
}

\author{
Fatma MEN KILINÇ $^{1^{*}} \quad$ Mesut KAYA ${ }^{2}$ Tuğba EMEKCỉ ${ }^{3}$ Deniz Uğur CENGIZZ ${ }^{4}$ \\ 1.Başkent University, Faculty of Medicine ENT Clinic, Department of Audiology, Ankara, Turkey \\ 2.Private ENT Clinic, Ankara, Turkey \\ 3.Necmettin Erbakan University, Faculty of Medicine ENT Clinic, Konya, Turkey \\ 4.İnönü University, Faculty of Health Science, Department of Audiology, Malatya, Turkey
}

\begin{abstract}
Background: In the occurrence of motion sickness, the functioning of the vestibular system in harmony has an important role.

Aim/Objective: To evaluate the effectiveness of the vestibular system in individuals with motion disease with the Video Head Impulse Test (vHIT) device, which evaluates the vestibulo-ocular reflex (VOR).

Materials and Methods: Thirty individuals with motion sickness ( 21 females, 9 males) and 30 healthy individuals (11 females, 19 males) without any vestibular complaints were examined with the vHIT device. All participants were older than 18 years.

Results: It was observed that VOR gain values of the individuals with motion disease were significantly lower than those of the healthy individuals. Asymmetry values of the individuals with motion disease in all semicircular canals were higher than those of the healthy individuals; however, this difference was statistically significant only in the anterior semicircular canal.

Conclusion and Significance: The observation of low gain values in all semicircular canals in individuals with motion sickness suggested that individuals with motion sickness may experience dysfunction in the sensory organelles in the vestibular system. Studies with larger case series and audiological test batteries will help us better understand motion sickness.
\end{abstract}

Keywords: Motion sickness; vestibular; vHIT

DOI: $10.7176 / \mathrm{JHMN} / 79-05$

Publication date:August $31^{\text {st }} 2020$

\section{Introduction}

Motion sickness appears as a problem in healthy individuals during sea voyage, air/land travel, or an unfamiliar movement. With new technological developments, space travels and virtual reality activities may also cause motion sickness (BİLGEN and KİRAZLI). Motion sickness can be seen with symptoms such as nausea, vomiting, drowsiness, imbalance, paleness, headache, and cold sweating that appear temporarily during the individual's movement or the movement of the visual environment (Yates et al., 1998).

In the occurrence of motion sickness, the functioning of the vestibular system in harmony has an important role. Motion sickness mostly occurs in individuals with a healthy vestibular system when vestibular stimuli and visual stimuli do not function in harmony or due to unexpected/unfamiliar vestibular stimuli. The absence of motion sickness in individuals with bilateral vestibular system dysfunction is the best evidence for this outcome (Cheung et al., 1991).

Although many theories about motion sickness have been proposed to date, none have been able to fully explain the underlying mechanism. The hypothesis proposed by Reason and Brand (Reason and Brand, 1975), named as "perceptual conflict", has been commonly accepted. According to this theory, motion sickness stems from perceptual signals that contradict with the ones expected by the brain, which occurs in situations where motion or visual environment is unstable. The postural instability hypothesis proposed by Stoffreng and Riccio (Stoffregen and Smart Jr, 1998) is based on the coordinated stabilization of body components. Another theory, the neural incompatibility theory, is the result of comparing the "neural memory" containing past experiences by processing the stimuli from the vestibular, visual, and proprioceptive system in the parietal cortex, vestibular nuclei, and cerebellum. The occurrence of a conflict in this comparison is called "neural mismatch". With the occurrence of this neural mismatch, autonomic symptoms appear in motion sickness patients through the stimulation of the vestibulo-autonomic reflex (Shahal et al., 1999). In another theory regarding the formation of motion sickness, the area that is held responsible is the brain stem. Lateral medullary reticular formation and nucleus tractus solitarius, which are activated during the vomiting reaction, are said to be effective in homeostasis, which is associated with the respiratory system and the cardiovascular system in addition to gastrointestinal connections. (Yates et al., 1998).

The relationship of motion disease with the vestibular system has been demonstrated in many studies (Shahal et al., 1999, Shupak et al., 1990). It has been observed that, in individuals with motion sickness symptoms, compared to healthy individuals, statistically significant hyperactive vestibular functions were obtained in the caloric test results (BİLGEN and KİRAZLI), an increase in the latencies of the waves was obtained in the VEMP 
(Vestibuler Evoked Myogenic Potential) test (Trinus, 1988), and increased asymmetry rates were observed (Xie et al., 2012). vHIT is a new vestibular test battery. It provides the opportunity to compare with VOR gains and asymmetry rates of six semicircular canals. Our hypothesis is to observe a possible decrease in vestibular functions in evaluations with vHIT in patients with motion sickness, as in other vestibular test batteries.

The aim of this clinical study was to evaluate the semicircular canal functions using the vHIT, a new and complementary test battery, in patients diagnosed with motion sickness and presented to our clinic.

\section{Materials and Methods}

This research was planned as a prospective study. The study included patients who were presented to local university hospital, Audiology and Speech Disorders Clinic between January 2016 and March 2016. University Institute of Health Sciences Non-Interventional Clinical Research Ethics Committee (Decision number: 2016: 99950669/85) approved the study. The study included 30 individuals diagnosed with motion sickness based on routine ENT examinations and 30 healthy individuals. All participants were older than 18 years. Healthy individuals did not have any neurological and systemic disorders including hearing and balance problems. Inclusion criteria required for the subjects with Motion sickness: No histories of significant head trauma, neurological disease (e.g Parkinson's, post-polio syndrome, diabetic neuropathy), visual impairment not correctable with lenses, musculoskeletal impairments, persistent symptoms of vertigo, or hearing problems.

In our study, a vHIT (Otometrics ICS Impulse A/S Taastrup, Denmark) device was used. For each canal, 20 head-thrust movements were applied. VOR responses created as a result of the evaluations made with this test can be measured with a computer and a report can be obtained as a graphic. Head and eye movement curves for each canal, a regression curve showing the change in time in the velocity of the head thrust movement, gain values, a circular gain chart in which canal gains can be compared, and another chart showing the directions of vertical head movements. In addition, gain values and anterior, posterior, and lateral asymmetry values of all 6 semicircular canals are included in this report. The ideal VOR gains value obtained by comparing the acceleration of the movements of the eyes and the head in the opposite direction during the vHIT test and should be 1. Abnormal test result was considered as gain value less than 0.76, greater than 1.18 (Mossman et al., 2015). Normalization studies of the posterior and anterior SSC have also been performed and their normal values are expected to be close to 1 (MacDougall et al., 2013, Weber et al., 2008). In addition, in a vHIT result of a normal individual, the wave morphology should be symmetrical, there should be no refixation saccades and the asymmetry value should be below 7\% (Mossman et al., 2015). In our study, these VOR gain and asymmetry values are considered as normal.

The data were analyzed using the SPSS v.22 statistical program. The groups are independent and the number of individuals in both groups is 30 or more. The Kolmogorov-Smirnov test was used to test whether the data was normally distributed or not. The significance level of the test was determined to be 0.05 , and it was accepted that the data were normally distributed when the $p$ value was higher than 0.05 . The data were assumed to be not distributed normally when the $\mathrm{p}$ value was less than 0.05 . In the test of the homogeneity of variances, the homogeneity of variances test was applied and the level of significance was set at 0.05 . In the homogeneity test of variances, it was assumed that the variances were homogeneous when the $p$ value was greater than 0.05 . Similarly, in cases where the $p$ value was less than 0.05 , variances were considered to be non-homogeneous. According to the homogeneity test of variances results, it was determined that the gain values were not homogeneous between the groups in any canal $(p<0.05)$. As a result, the Mann Whitney $U$ test was applied to test whether there is a significant difference between groups in terms of VOR gains.

\section{Results}

In the present study, $63.33 \%(n=19)$ of the control group $(n=30)$ were male and $36.67 \%(n=11)$ were female, and $30 \%(n=9)$ of the motion sickness group $(n=30)$ were male and $70 \%(n=21)$ were female. The average age of the healthy group was 24.77 years and the average age of the motion sickness group was 29.00 years (Table 1). Table 1. Distribution of Demographic Variables $(n=60)$

\begin{tabular}{llllll}
\hline & \multicolumn{3}{l}{ Control group } & \multicolumn{2}{l}{ Motion sickness } \\
& & $\mathrm{N}$ & $\%$ & $\mathrm{~N}$ & $\%$ \\
\multirow{2}{*}{ Sex } & 19 & 63.33 & 9 & 30.0 \\
\hline \multirow{2}{*}{ Male } & 11 & 36.67 & 21 & 70.0 \\
\hline Female & Mean & SD & Mean & SD \\
\hline
\end{tabular}

The distribution of the individuals included in the study based on demographic variables is summarized.

In the present study, we have measured VOR gain and VOR gain asymmetry value in individuals with and without motion sickness. 
The right lateral superior semicircular canal (SSC) VOR gain mean $0.75 \pm 0.408$ of the motion sickness group was statistically lower than that of the healthy group $0.97 \pm 0.153(\mathrm{p}<0.05)$. Similarly, the left lateral SSC VOR gain mean $0.73 \pm 0.329$ of the motion sickness group was significantly lower than that of the control group $0.93 \pm$ $0.084(\mathrm{p}<0.05)$.

The right anterior SSC VOR gain mean $0.65 \pm 0.283$ of the motion sickness group was statistically lower than that of the control group $0.90 \pm 0.133$ ( $\mathrm{p}<0.05$ ). Similarly, the left anterior SSC VOR gain mean $0.82 \pm 0.247$ of the motion sickness group was statistically lower than that of the control group $0.94 \pm 0.1123(\mathrm{p}<0.05)$.

The mean posterior SSC VOR gain mean $0.52 \pm 0.327$ of the motion sickness group was statistically lower than that of the control group $0.88 \pm 0.130(\mathrm{p}<0.05)$. Similarly, the left posterior SSC VOR gain mean $0.58 \pm$ 0.360 of the motion sickness group was statistically lower than that of the control group $0.90 \pm 0.204(p<0.05)$ (Table 2).

Table 2. VOR Gain Values of the control and Motion sickness groups

\begin{tabular}{lllll}
\hline VOR Gains & & Control Group & Motion sickness Group & p Value \\
\hline \multirow{2}{*}{ Lateral } & Right & $0.97( \pm 0.153)$ & $0.75( \pm 0.408)$ & 0.047 \\
\cline { 2 - 5 } & Left & $0.93( \pm 0.084)$ & $0.73( \pm 0.329)$ & 0.011 \\
\hline \multirow{2}{*}{ Anterior } & Right & $0.90( \pm 0.133)$ & $0.65( \pm 0.238)$ & 0.000 \\
\cline { 2 - 5 } & Left & $0.94( \pm 0.123)$ & $0.82( \pm 0.247)$ & 0.045 \\
\hline \multirow{2}{*}{ Posterior } & Right & $0.88( \pm 0.130)$ & $0.52( \pm 0.327)$ & 0.000 \\
\cline { 2 - 5 } & Left & $0.90( \pm 0.204)$ & $0.58( \pm 0.360)$ & 0.000
\end{tabular}

The VOR gain values of the individuals included in the study are summarized. $(\mathrm{p}<0.05)$

Although the lateral and posterior SSC gain asymmetry values of the motion sickness group were higher than those of the control group, the difference was not statistically significant $(p>0.05)$. The anterior SSC gain asymmetry value of the motion sickness group was statistically higher than that of the control group $(p<0.05)$ (Table 3).

Table 3. VOR gain asymmetry values of the control and Motion sickness groups

\begin{tabular}{llll}
\hline Asymmetry Values & Control Group & Motion sickness & $\mathrm{p}$ Value \\
\hline Lateral & $8.10( \pm 9.617)$ & $22.13( \pm 25.666)$ & 0.085 \\
\hline Anterior & $12.00( \pm 8.497)$ & $33.80( \pm 26.385)$ & 0.004 \\
\hline Posterior & $13.84( \pm 10.823)$ & $32.10( \pm 32.604)$ & 0.166 \\
\hline
\end{tabular}

VOR gain asymmetry values of individuals included in the study are summarized. $(p<0.05)$

\section{Discussion}

In our study, we compared SSC functions with a vHIT device in healthy individuals and those with motion sickness complaints It was hypothesized that there may be a possible decrease in vestibular function in evaluations with vHIT, as in other vestibular test batteries. Our hypothesis was supported. In our study, when we compared the VOR gains between the right ear lateral SSC values of the study group individuals $0.75 \pm 0.408$ and the control group individuals $0.97 \pm 0.153$, and the VOR gains between the left ear lateral SSC values of the individuals in the study group $0.73 \pm 0.329$ and in the control group $0.93 \pm 0.084$ are significantly different against the study group $(\mathrm{p}<0.05)$. The anterior canal asymmetry values $33,80 \pm 26,385$ of the individuals in the study group were higher than the control group $12,00 \pm 8,497$ and this difference was statistically significant $(\mathrm{p}<0.05)$. In other channels, although the difference was not statistically significant, the asymmetry values of all channels were higher than $22 \%$.

Neupane et al. (Neupane et al., 2018) reported that all SSC VOR gains of the individuals with motion sickness were found to be statistically lower than those of the healthy individuals, which is in line with the results of the present study. Studies showing the decrease in VOR gains in individuals with peripheral vestibular pathology indicate the effect of motion sickness on the peripheral system (Weber et al., 2008, MacDougall et al., 2013).

In our study, in accordance with the work of Neupane et al. (Neupane et al., 2018), the asymmetry rates of the individuals with motion sickness were higher than those of the healthy individuals. However, the asymmetry rates of the anterior canals of the individuals with motion sickness were statistically higher than those of the healthy individuals, while no statistical difference was found in other canals. Achieving high asymmetry rates in individuals with motion sickness is explained by the intra-sensory conflict. Intra-sensory conflict arises from the difference in neural inputs from different planes to the cortical equilibrium areas. Vomiting symptom can be seen with this processing difference (Yates et al., 1998). In addition, the asymmetry rate suggests vestibular disorders 
as an important parameter in determining SSC pathologies (Neupane et al., 2018). In a study in which asymmetry rates were evaluated using a Scleral search coil device, asymmetry rates were found between $22 \%$ and $65 \%$ in groups with vestibular pathology (MacDougall et al., 2013, Weber et al., 2008). The fact that the asymmetry rates of the individuals with motion sickness in our study were between these values may suggest a vestibular disorder.

In a study comparing individuals with motion sickness and healthy controls, hyperactive vestibular function was obtained by applying the bithermal water caloric test (BILGEN and KİRAZLI). As hyperactive vestibular functions are generally seen in central nerve system disorders, motion sickness is thought to affect the central system (Shahal et al., 1999). Although the lateral and posterior SSC gain asymmetry values of the motion sickness group were higher than those of the control group, the difference was not statistically significant $(p>0.05)$. The anterior SSC gain asymmetry value of the motion sickness group was found to be statistically higher than that of the control group $(\mathrm{p}<0.05)$. In another study, the phase and VOR gains at the lateral canals at the frequencies of $0.01,0.02,0.04,0.08$, and $0.16 \mathrm{~Hz}$ were evaluated with a rotational chair. At higher frequencies $(0.04-0.16 \mathrm{~Hz})$, the phase was significantly lower compared to lower frequencies (0.01-0.02 Hz). Also, there was no correlation between motion sickness level and VOR gain (Clement and Reschke, 2018). When the VOR gains of the lateral canals obtained by the vHIT in the present study and the other audiological test results evaluating the lateral canals at different frequencies in the literature were considered, motion sickness is thought to have different effects on the vestibular system at different frequencies (Neupane et al., 2018, Clement and Reschke, 2018, BİLGEN and KİRAZLI). In another study, the cVEMP test was administered in individuals with seasickness and healthy individuals. While the majority of the individuals with seasickness did not yield a wave, the rate of asymmetry was determined as $35 \%$ in the waves obtained. This result was explained by the neural mismatch theory and it was thought that an adaptation effort occurred in the vestibular system (Boldingh et al., 2011).

\section{Considerations/limitations}

Since audiological test batteries are not effective in diagnosis alone, each test battery should be used as complementary to the diagnosis. Today, it is considered that further studies are needed with new test batteries in order to fully identify motion sickness, whose physiopathology has not been completely clarified. In motion sickness assessment, vHIT, VEMP, VNG, and posturography devices should be used and evaluated together to evaluate the peripheral and central vestibular system together and to obtain more objective results.

\section{Acknowledgements}

The authors are grateful to all participants in this study.

\section{Disclosure statement}

No potential conflict of interest was reported by the authors.

\section{References}

BİLGEN, C. \& KİRAZLI, T. Hareket Hastalığında Elektronistagmografinin Değeri.

BOLDINGH, M. I., LJØSTAD, U., MYGLAND, Å. \& MONSTAD, P. 2011. Vestibular sensitivity in vestibular migraine: VEMPs and motion sickness susceptibility. Cephalalgia, 31, 1211-1219.

CHEUNG, B., HOWARD, I. \& MONEY, K. 1991. Visually-induced sickness in normal and bilaterally labyrinthine-defective subjects. Aviation, space, and environmental medicine.

CLEMENT, G. \& RESCHKE, M. F. 2018. Relationship between motion sickness susceptibility and vestibuloocular reflex gain and phase. Journal of Vestibular Research, 28, 295-304.

MACDOUGALL, H. G., MCGARVIE, L. A., HALMAGYI, G. M., CURTHOYS, I. S. \& WEBER, K. P. 2013. The video head impulse test (vHIT) detects vertical semicircular canal dysfunction. PloS one, 8 .

MOSSMAN, B., MOSSMAN, S., PURDIE, G. \& SCHNEIDER, E. 2015. Age dependent normal horizontal VOR gain of head impulse test as measured with video-oculography. Journal of Otolaryngology-Head \& Neck Surgery, 44, 29.

NEUPANE, A. K., GURURAJ, K. \& SINHA, S. K. 2018. Higher asymmetry ratio and refixation saccades in individuals with motion sickness. Journal of the American Academy of Audiology, 29, 175-186.

REASON, J. T. \& BRAND, J. J. 1975. Motion sickness, Academic press.

SHAHAL, B., NACHUM, Z., SPITZER, O., BEN - DAVID, J., DUCHMAN, H., PODOSHIN, L. \& SHUPAK, A. 1999. Computerized dynamic posturography and seasickness susceptibility. The Laryngoscope, 109, 19962000.

SHUPAK, A., KEREM, D., GORDON, C., SPITZER, O., MENDELOWITZ, N. \& MELAMED, Y. 1990. Vestibulo-ocular reflex as a parameter of seasickness susceptibility. The Annals of otology, rhinology, and laryngology, 99, 131-136.

STOFFREGEN, T. A. \& SMART JR, L. J. 1998. Postural instability precedes motion sickness. Brain research bulletin, 47, 437-448. 
TRINUS, K. 1988. Vestibular evoked potentials-a new method for study of the combined effects of environmental factors. Recent advances in researches on the combined effects of environmental factors. Proceedings of the Third IntemationalConference on the combined Effects of Environmental Factors (ICCEF 88), 15-18.

WEBER, K., AW, S., TODD, M., MCGARVIE, L., CURTHOYS, I. \& HALMAGYI, G. 2008. Head impulse test in unilateral vestibular loss: vestibulo-ocular reflex and catch-up saccades. Neurology, 70, 454-463.

XIE, S.-J., CHEN, W., JIA, H.-B., WANG, Z.-J., YAO, Q. \& JIANG, Y.-Y. 2012. Ocular vestibular evoked myogenic potentials and motion sickness susceptibility. Aviation, space, and environmental medicine, 83, 1418.

YATES, B., MILLER, A. \& LUCOT, J. 1998. Physiological basis and pharmacology of motion sickness: an update. Brain research bulletin, 47, 395-406. 